\title{
Force-sensitive Electronic Probe
}

National Cancer Institute

\section{Source}

National Cancer Institute. Force-sensitive Electronic Probe. NCI Thesaurus. Code C94570.

A periodontal probe that uses a standardized pressure to measure the depth of periodontal pockets, recession, or attachment levels. 